\title{
Socioeconomic differences in self-rated health among women: a comparison of St. Petersburg to Estonia and Finland
}

\author{
Tatiana Dubikaytis ${ }^{1}$, Tommi Härkänen², Elena Regushevskaya ${ }^{2 *}$, Elina Hemminki², Elina Haavio-Mannila ${ }^{3}$, \\ Made Laanpere ${ }^{4}$, Olga Kuznetsova ${ }^{1}$ and Seppo Koskinen ${ }^{2}$
}

\begin{abstract}
Introduction: Social determinants of health have not been intensively studied in Russia, even though the health divide has been clearly demonstrated by an increased mortality rate among those with low education. A comparative analysis of social health determinants in countries with different historical and economic backgrounds may provide useful evidence for addressing health inequalities. We aimed to assess socioeconomic determinants of self-rated health in St. Petersburg as compared to Estonia and Finland.

Methods: Data for women aged 18-44 were extracted from existing population-based surveys and analysed. In St. Petersburg the data were originally collected in 2003 (response rate 68\%), in Estonia in 2004-2005 (54\%), and in Finland in 2000-2001 (86\%). The study samples comprised 865 women in St. Petersburg, 2141 in Estonia and 1897 in Finland.

Results: Self-rated health was much poorer in St. Petersburg than in Estonia or Finland. High education was negatively associated with poor self-rated health in all the studied populations; it was (partially) mediated via health behaviour and limiting long-term illness only in Estonia and Finland, but not in St. Petersburg. High personal income and employment did not associate with poor self-rated health among St. Petersburg women, as it did in Estonia and Finland. In St. Petersburg housewives rather than employed women had better self-rated health, unlike the two other areas.

Conclusion: Women's self-rated health in St. Petersburg varied similarly by education but differently by income and employment as compared to Estonia and Finland. Education is likely the most meaningful dimension of women's socioeconomic position in St. Petersburg. More research is needed to further clarify the pathways between socioeconomic position and health in Russia.
\end{abstract}

Keywords: Health status, Education, Income, Health behaviour, Health disparities, Russia, Women

\section{Introduction}

In many European countries growing awareness of health inequalities within and between countries has resulted in intensive research of the social health gradient. It has been shown that the influence of socioeconomic position on health is mediated by diverse mechanisms that operate on individual and societal levels [1-6]. The pattern of health inequality within countries can be shaped by national health promotion policy, social security regulations, and the economic and political environment. Therefore

\footnotetext{
* Correspondence: elena.regushevskaya@thl.fi

${ }^{2}$ The National Institute for Health and Welfare (THL), P.O. Box 30, Helsinki Fl-00271, Finland

Full list of author information is available at the end of the article
}

comparisons across countries of health inequality may yield a better understanding of the complex interrelationships between socio-economic position and health.

Self-rated health is a widely used measure for population health status. It correlates with physical health, functional capacity, psychological well-being, and is a significant predictor of morbidity, mortality and health care utilization [7-9]. Analysis of the correlations between socioeconomic position and self-rated health is often used to assess pathways in the development of health disparities.

Health inequalities in post-Soviet Russia are of particular interest. Economic and social changes in the 1990s along with an accumulation of social health hazards over 
a life-course resulted in a deterioration in population health, especially of those who had a less advantaged socioeconomic position [10,11]. Still, in Russia the social determinants of self-rated health have been studied mainly by international researchers and the data are limited. Studies have been conducted in different parts of the country (Kazan, Taganrog, Novosibirsk, Moscow and Pitkäranta) [12-17] and only a few have analysed the entire Russian population $[10,18,19]$. There are conflicting results on the socioeconomic variations in self-rated health: education was associated with better health in women and men in Taganrog [11] and only among women in Pitkäranta [11]; the association was substantial in Moscow men and weak among Moscow women [12]; household income correlated with better health in women, but not among men in Pitkäranta [11].

The contribution of health behaviour to socioeconomic self-rated health differences is unclear in Russia. An association between lifestyle-related risk factors and educational difference in cardiovascular mortality and morbidity, similar to that in western countries, has been found among Russian men, but among women the role of these factors was less obvious [20,21]. Therefore it is of interest to analyse lifestyle pathways between socioeconomic position and health among Russian women. Available statistics show that the overall level of women's health as measured by deaths is much worse in Russia than in Finland [22]; Estonian women fall somewhere between [23].

The aim of the present study is to describe the socioeconomic self-rated health gradient and to assess the contribution of behavioural risk factors to this gradient among reproductive age women in St. Petersburg, Russia's second biggest city, and to compare the results to two neighbouring countries: Estonia and Finland. Socioeconomic position was assessed using education, personal income and employment status. In addition we considered family structure and long-standing illness as potential confounders [24].

Estonia is another post-Soviet country and Finland is geographically very close to St. Petersburg. Earlier studies concerning self-rated health in Finland [25] and cross national comparisons [26,27] showed that self-perceived health was poorer in Estonia than in Finland. In both countries women with low education had poor self-rated health. In Estonia, but not in Finland economic inactivity was associated with poor self-rated health [27].

\section{Methods}

In St. Petersburg an anonymous survey was conducted in 2003-2004 in two areas of the city [28,29]. The women were given the questionnaire to be completed during a health examination or at home and either collected or mailed later. A random sample of 2501 women aged 18-44 was drawn; 1719 women were contacted and $1152(67 \%)$ participated in the survey. For a detailed description see earlier reports [28,29].

In Estonia, a postal survey was conducted in 20042005 [30]. A random sample $(n=5190)$ of all women aged 16-44 was drawn, anonymous questionnaires were posted (3472 in Estonian and 1718 in Russian language) and 2735 (54\%) completed questionnaires were received.

In Finland the Health 2000 Health Examination Survey was conducted in 2000-2001 [31]. A two-stage cluster sample $(\mathrm{N}=10000)$ that was representative of the population aged 18 or older was drawn. The response rate was $80 \%$ for young adults (aged 18 to 29 years) and $89 \%$ for those aged 30 years or older [32]. The sample contained 2266 women aged 16-44, of which $86 \%$ participated. Most of the variables used in this study were collected in the interview at the respondent's home. Information on income was obtained from a national register and BMI was based on measured weight and height.

The study questionnaires were prepared jointly by Finnish, Russian and Estonian researchers for the St. Petersburg and Estonian surveys with the aim of ensuring maximum comparability with the Finnish Health 2000 Survey, regardless of the different language structures. The questionnaires in St. Petersburg and Estonia were constructed at the same time and were similarly formulated using the questions from the Finnish survey as a model [28].

Our data analysis was restricted to females aged 18-44. We excluded pensioners (for illness or handicap, 3 in St. Petersburg, 22 in Estonia, 22 in Finland) and women with missing values in any of the studied variables. The proportion of women excluded was higher in St. Petersburg (24\%) compared to Estonia (15\%) and Finland (3\%). The total numbers of women whose data were used in the analyses were 865 in St. Petersburg, 2141 in Estonia and 1897 in Finland.

\section{Variables}

All variables with the exception of income data in Finland were based on self-reported information. The outcome measure was self-rated health, which was obtained with the question: "How satisfied are you with your health?" with a five-point response scale ( 1 - very satisfied; 2 - satisfied; 3 - neither satisfied nor dissatisfied; 4 - dissatisfied; and 5 - very dissatisfied). Self-reported limiting long-term illnesses were obtained with the question "Do you have any permanent or chronic illness or any defect, difficulty or injury that reduces your working capacity and functional ability: Yes/No? If yes, please specify the exact medical condition". The reported limiting diseases were classified into four groups: mental, cardio-vascular, trauma \& musculoskeletal diseases and other diseases, based on our awareness that those conditions could be differently distributed across socioeconomic groups. 
Level of education was measured by the total number of study years completed. We used a categorisation that was applied previously in a comparative study using the same data [28]. In St Petersburg and Estonia education was categorised: $<11,11-13,14-16$ and $>16$ years of education; In Finland: $\leq 10,11-12,13-15$, and $\leq 16$ years. Personal income was classified into four quartiles; in Russia and Estonia income was self-reported, but in Finland it was obtained from a national tax authority. Family structure was classified based on a question about marital status and number of children under 18 years of age living with the respondent. Four categories were created: living with a partner (married or cohabiting) and a child/children; living only with partner; living without partner/children; living only with a child/children. Employment status was classified into four categories: employed, student, unemployed, or housewife, based on the following question: "What is your main economic activity: employed, unemployed, housewife, full-time student, retired, not employed or others?" Occupation was asked in each survey,

Table 1 Age-adjusted means, proportions or distributions of the explanatory variables by country

\begin{tabular}{|c|c|c|c|c|}
\hline & $p$-value ${ }^{c}$ & St. Petersburg & Estonia & Finland \\
\hline Number of women ${ }^{a}$ & & 865 & 2141 & 1897 \\
\hline Mean income per month (in euro) & $p<0.001$ & $130,1 \pm 5,5$ & $243,0 \pm 4,3$ & $874,9 \pm 14,4$ \\
\hline Education, yrs & $p<0.001$ & & & \\
\hline$<11(<10)^{\mathrm{b}}$ & & 5 & 6 & 6 \\
\hline $11-13(10-12)$ & & 41 & 45 & 27 \\
\hline $14-16(13-15)$ & & 42 & 35 & 38 \\
\hline $17+(16+)$ & & 11 & 13 & 30 \\
\hline Family, \% & $p<0.001$ & & & \\
\hline Partner \& child/children & & 36 & 45 & 44 \\
\hline Only partner, no child/children & & 24 & 19 & 24 \\
\hline No partner no child/children & & 29 & 22 & 23 \\
\hline Only child/children, no partner & & 11 & 13 & 9 \\
\hline Employment, \% & $p<0.001$ & & & \\
\hline Employed & & 76 & 56 & 64 \\
\hline Student & & 11 & 24 & 16 \\
\hline Unemployed & & 3 & 4 & 9 \\
\hline Housewives & & 10 & 15 & 11 \\
\hline BMI, \% & $p<0.001$ & & & \\
\hline Under 20 & & 26 & 26 & 16 \\
\hline $20-24.9$ & & 49 & 51 & 52 \\
\hline $25-29.9$ & & 16 & 17 & 22 \\
\hline $30+$ & & 9 & 7 & 10 \\
\hline Smoking, \% & $p<0.001$ & & & \\
\hline Never & & 41 & 52 & 58 \\
\hline In past & & 14 & 18 & 14 \\
\hline Occasional & & 14 & 8 & 5 \\
\hline Current & & 31 & 22 & 23 \\
\hline \multicolumn{5}{|l|}{ Chronic diseases, $\%$} \\
\hline Yes vs. no & $p<0.001$ & 38 & 24 & 29 \\
\hline Mental diseases, $\%$ & $p<0.01$ & 1 & 1 & 2 \\
\hline Trauma and musculoskeletal, $\%$ & $p<0.001$ & 6 & 7 & 9 \\
\hline Cardio-vascular diseases, \% & $p<0.001$ & 6 & 4 & 2 \\
\hline Other diseases, \% & $p<0.001$ & 25 & 13 & 16 \\
\hline
\end{tabular}

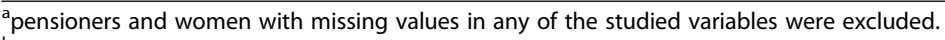

${ }^{b}$ years of education in brackets corresponds to Finnish population.

'Wald test for comparing countries. 
but we could not categorise it in a comparable way in the three areas, and so occupation was not included in the analysis.

Behavioural factors used in the analysis included smoking and body mass index (BMI), which was calculated from weight and height. BMI indicates a misbalance between calorific consumption and spending, which can reflect a lack of physical activity and/or unhealthy diet. Smoking was defined based on the question "Have you ever smoked regularly at least one cigarette (cigar or pipe tobacco) daily for at least one year?" with further clarification which allowed for four groups: never, in past, occasional and current. We used selfreported height and weight in the Russian and Estonian survey while in Finland, height and weight were measured. BMI was classified into four categories: low weight (under 20), normal weight (20-24), overweight (25-29) and obesity (30 and over).

\section{Statistical analysis}

Associations between the explanatory variables and selfrated health were analysed using ordinal logistic regression models, with estimated cumulative odds ratios (COR) presented. The outcome self-rated health was modelled as an ordinal outcome with five categories ( 1 as the best and 5 as the poorest), into cumulative odds ratios (COR). The COR represents the proportional change in the odds for a subject being in categories $1, \ldots, \mathrm{k}$ vs. in categories $\mathrm{k}+1, \ldots, 5$ (for any $1<=\mathrm{k}<=4$ ). If there were only two outcome categories, then the model would be the standard logistic regression model giving odds ratios. We did not dichotomize the outcome as information would have been lost as well as statistical power.

We have assessed the influence of the variables in the possible causal pathway using the elaboration analysis. If a variable of interest, for example education, has influence on the outcome, then the adjustment for intermediate variables such as the behavior risk factors or chronic diseases does not remove the association of education and the outcome. These intermediate variables have been added group-wise following the assumed causal pathway. First the socio-economic position, second the behavior risk factors and third the chronic diseases. In model I, adjustment for age was applied; in model II socioeconomic position factors were mutually adjusted; in model III smoking and BMI were additionally incorporated; and in model IV diseases were additionally included. The analysis was performed with STATA 11.0 (StataCorp, 2009). The age-adjusted distributions of the explanatory variables by country were estimated with logistic regression and the predictive margins method [33]. The country differences were tested with the Wald test. The age- adjusted distributions were based on the predictive margins method [33]. The significance of interactions between the country and the explanatory variables was tested with the Wald test to assess the possible effect-modification of the country.

\section{Results}

Women's background characteristics varied in the three areas (Table 1). St. Petersburg and Finnish women were most different from each other and Estonian women were in between. Mean income in Euros was notably higher in Finland than in Estonia and St. Petersburg. Smoking was more common in St. Petersburg, as were longstanding limiting illnesses.

Self-rated health varied notably by area (Table 2 ). The proportion of women who rated their health to be poor was notably higher in St. Petersburg than in Finland; women in Estonia were in between. The estimated CORs for the associations between the explanatory variables and self-rated health are presented in Tables 3, 4 and 5, for the St. Petersburg, Estonian and Finnish populations, respectively.

\section{Variation in self-rated health by socioeconomic position} In the age-adjusted models we found a negative association between high education and poor SRH in all studied populations $(\mathrm{COR}=0.42$ for St. Petersburg women, $\mathrm{COR}=0.38$ for Estonian women and $\mathrm{COR}=0.35$ for Finnish women, Figure 1 and Tables 3, 4 and 5). Adjustment for age did not change the association between education and poor SRH, but adjustment for all factors removed the statistical significance in Estonia (Figure 1).

Adjusting for age, personal income and SRHs did not correlate strongly in St. Petersburg in contrast to Estonia and Finland, Figure 2. Adjustment for all factors (Model IV) removed the statistical significance of income in Finland, whereas in Estonia the significance remained. The interaction between country and personal income was significant (data not shown).

Compared to employed women, unemployment was associated with poor SRH in Estonia and Finland, but not in St. Petersburg, Figure 3. After adjusting for all background characteristics the statistical significance of the association in Estonia and Finland vanished.

Table 2 Distribution of women by their age-adjusted self-rated health by country, \%

\begin{tabular}{llll}
\hline & St. Petersburg & Estonia & Finland \\
\hline (Number of women) & $(865)$ & $(2141)$ & $(1897)$ \\
Very satisfied & 6 & 16 & 56 \\
Satisfied & 30 & 48 & 36 \\
Neither satisfied nor dissatisfied & 32 & 23 & 6 \\
Dissatisfied and very dissatisfied & 32 & 12 & 2 \\
Total & 100 & 100 & 100 \\
\hline
\end{tabular}


Table 3 Cumulative OR with $95 \% \mathrm{Cl}$ for self-rated health ( 1 = best health and $5=$ poorest health) by socioeconomic, behaviour factors and longstanding illness among women in St. Petersburg

\begin{tabular}{|c|c|c|c|c|}
\hline & Model I age-adjusted $^{\mathrm{a}}$ & $\begin{array}{l}\text { Model II + SEP } \\
\text { adjusted }^{b, c}\end{array}$ & Model III + behavior risk factors & Model IV + chronic diseases ${ }^{\mathrm{b}, \mathrm{e}}$ \\
\hline Education & $p^{f}<0.01$ & $p^{f}<0.05$ & $p^{f}<0.10$ & $p^{f} n s$ \\
\hline$<11$ yrs & 1.00 & 1.00 & 1.00 & 1.00 \\
\hline $11-13$ yrs & $0.67[0.41-1.09]$ & $0.65^{\#}[0.39-1.07]$ & $0.64[0.39-1.06]$ & $0.65[0.38-1.12]$ \\
\hline $14-16$ yrs & $0.51^{* *}[0.32-0.82]$ & $0.52^{*}[0.31-0.85]$ & $0.54^{*}[0.33-0.89]$ & $0.55^{*}[0.32-0.96]$ \\
\hline $17+y r s$ & $0.42^{* *}[0.23-0.77]$ & $0.40^{* *}[0.22-0.75]$ & $0.46^{*}[0.25-0.87]$ & $0.46^{*}[0.23-0.91]$ \\
\hline Personal income & $p^{f}$ ns & $p^{f} n s$ & $p^{f} n s$ & $p^{f} n s$ \\
\hline Lowest quartile & 1.00 & 1.00 & 1.00 & 1.00 \\
\hline 2nd quartile & 1.17 [0.80-1.69] & $0.87[0.56-1.36]$ & $0.88[0.56-1.38]$ & 0.87 [0.55-1.39] \\
\hline 3rd quartile & $1.00[0.69-1.45]$ & $0.77[0.49-1.21]$ & $0.82[0.52-1.30]$ & $0.79[0.50-1.26]$ \\
\hline Highest quartile & $0.85[0.60-1.21]$ & $0.69[0.44-1.08]$ & $0.69[0.44-1.08]$ & $0.65^{\#}[0.41-1.04]$ \\
\hline Employment & $p^{f} n s$ & $p^{f}<0.05$ & $p^{f}<0.10$ & $p^{f}<0.10$ \\
\hline Employed & 1.00 & 1.00 & 1.00 & 1.00 \\
\hline Student & $0.77[0.49-1.19]$ & $0.74[0.44-1.23]$ & $0.78[0.46-1.32]$ & $0.66[0.37-1.17]$ \\
\hline Unemployed & $1.32[0.72-2.42]$ & $1.04[0.54-2.01]$ & $1.04[0.54-2.02]$ & 1.09 [0.58-2.06] \\
\hline Housewives & $0.66^{\#}[0.42-1.03]$ & $0.47^{*}[0.27-0.83]$ & $0.48^{*}[0.27-0.86]$ & $0.50^{*}[0.28-0.89]$ \\
\hline Family structure & $p^{f} n s$ & $p^{f} n s$ & $p^{f} n s$ & $p^{f} n s$ \\
\hline Child \& partner & 1.00 & 1.00 & 1.00 & 1.00 \\
\hline Only partner & $1.01[0.74-1.38]$ & $1.00[0.72-1.38]$ & $0.89[0.64-1.25]$ & $0.97[0.68-1.38]$ \\
\hline No children no partner & $0.87[0.62-1.22]$ & $0.85[0.59-1.23]$ & $0.79[0.55-1.14]$ & $0.78[0.54-1.13]$ \\
\hline Only children & $0.98[0.67-1.44]$ & $0.90[0.61-1.34]$ & $0.92[0.62-1.37]$ & $0.84[0.55-1.28]$ \\
\hline Smoking & $p^{f}<0.10$ & & $p^{f} n s$ & $p^{f} n s$ \\
\hline Never & 1.00 & & 1.00 & 1.00 \\
\hline In past & $1.26[0.84-1.87]$ & & $1.19[0.78-1.80]$ & $1.23[0.80-1.90]$ \\
\hline Occasional & $1.04[0.74-1.47]$ & & $1.01[0.71-1.44]$ & $1.04[0.72-1.50]$ \\
\hline Current & $1.44^{*}[1.07-1.93]$ & & $1.31^{\#}[0.97-1.78]$ & $1.34^{\#}[0.98-1.82]$ \\
\hline BMI & $p^{f}<0.001$ & & $p^{f}<0.01$ & $p^{f}<0.05$ \\
\hline Under 20 & $1.24[0.90-1.72]$ & & $1.27[0.91-1.78]$ & $1.16[0.82-1.65]$ \\
\hline $20-24.9$ & 1.00 & & 1.00 & 1.00 \\
\hline $25-29.9$ & $1.66^{* *}[1.19-2.30]$ & & $1.63^{* *}[1.15-2.31]$ & $1.45^{*}[1.02-2.06]$ \\
\hline $30+$ & $2.44^{* *}[1.57-3.79]$ & & $2.33^{* * *}[1.48-3.67]$ & $1.95^{* *}[1.23-3.12]$ \\
\hline Mental illness & $p^{f} n s$ & & & $p^{f} n s$ \\
\hline Yes vs. no & $1.86[0.15-22.4]$ & & & $4.53[0.26-77.66]$ \\
\hline Trauma \& musculoskeletal & $p^{f}<0.001$ & & & $p^{f}<0.001$ \\
\hline Yes vs. no & $2.31^{* * *}[1.44-3.70]$ & & & $3.75^{* * * *}[2.27-6.18]$ \\
\hline Cardiovascular illness & $p^{f}<0.001$ & & & $p^{f}<0.001$ \\
\hline Yes vs. no & $2.79^{* * *}[1.86-4.19]$ & & & $4.49^{* * *}[2.91-6.92]$ \\
\hline Other diseases & $p^{f}<0.001$ & & & $p^{f}<0.001$ \\
\hline Yes vs. no & $3.15^{* * *}[2.40-4.14]$ & & & $4.47^{* * *}[3.31-6.04]$ \\
\hline
\end{tabular}

$\mathrm{SEP}=$ socioeconomic position, $\mathrm{BMI}=$ body mass index.

${ }^{\mathrm{a}}$ Model I SRH = age + one of the explanatory variables (education, personal income, employment status, family structure, BMI, smoking, chronic diseases).

${ }^{b}$ Elaboration analysis by adding a group of explanatory variables in the model allows one to assess the mediation effect of the group of new variables.

${ }^{c}$ Model II SRH = age + SEP variables (education + personal income + employment status + family structure).

${ }^{\mathrm{d}}$ Model III SRH = age + SEP variables + behaviour variables (BMI + smoking).

${ }^{\mathrm{e}}$ Model IV SRH = age + SEP variables + behaviour variables + chronic diseases.

fWald test for significance of the cumulative OR difference between the categories of the variable, ${ }^{\#} p<0.1 ;{ }^{*} p<0.05$; ${ }^{* *} p<0.01$; ${ }^{* * *} p<0.001 ;$ ns not significant. 
Table 4 Cumulative OR with $95 \% \mathrm{Cl}$ for self-rated health ( 1 = best health and $5=$ poorest health) by socioeconomic, behaviour factors and longstanding illness among women in Estonia ${ }^{a}$

\begin{tabular}{|c|c|c|c|c|}
\hline & Model I age-adjusted ${ }^{b, c}$ & Model II + SEP adjusted ${ }^{c, d}$ & $\begin{array}{l}\text { Model III + behavior } \\
\text { risk factors }{ }^{c, e}\end{array}$ & $\begin{array}{c}\text { Model IV + chronic } \\
\text { diseases }\end{array}$ \\
\hline Education & $p^{9}<0.001$ & $p^{9}<0.05$ & $\mathrm{p}^{\mathrm{g}} \mathrm{ns}$ & $p^{g} n s$ \\
\hline$<11$ yrs & 1.00 & 1.00 & 1.00 & 1.00 \\
\hline $11-13$ & $0.58^{*}[0.39-0.88]$ & $0.62^{*}[0.41-0.94]$ & $0.69^{\#}[0.46-1.05]$ & $0.77[0.49-1.19]$ \\
\hline $14-16$ & $0.45^{* * *}[0.29-0.68]$ & $0.53^{* *}[0.35-0.81]$ & $0.62^{*}[0.41-0.96]$ & $0.70[0.44-1.11]$ \\
\hline $17+$ & $0.38^{* * *}[0.24-0.61]$ & $0.52^{* *}[0.32-0.84]$ & $0.65^{\#}[0.40-1.08]$ & $0.73[0.43-1.22]$ \\
\hline Personal income & $\mathrm{p}^{9}<0.001$ & $\mathrm{p}^{9}<0.001$ & $p^{9}<0.001$ & $\mathrm{p}^{9}<0.01$ \\
\hline Lowest quartile & 1.00 & 1.00 & 1.00 & 1.00 \\
\hline 2nd quartile & $0.90[0.71-1.15]$ & $0.91[0.71-1.16]$ & $0.89[0.69-1.14]$ & $0.91[0.70-1.18]$ \\
\hline 3rd quartile & $0.69^{* *}[0.53-0.90]$ & $0.69^{*}[0.50-0.95]$ & $0.68^{*}[0.49-0.93]$ & $0.65^{* *}[0.47-0.90]$ \\
\hline Highest quartile & $0.50^{* * *}[0.39-0.65]$ & $0.53^{* * *}[0.39-0.73]$ & $0.52^{* * *}[0.38-0.71]$ & $0.58^{* *}[0.42-0.79]$ \\
\hline Employment & $\mathrm{p}^{9}<0.05$ & $p^{g} n s$ & $p^{g}$ ns & $p^{g} n s$ \\
\hline Employed & 1.00 & 1.00 & 1.00 & 1.00 \\
\hline Student & $1.04[0.81-1.33]$ & $0.89[0.67-1.19]$ & $0.91[0.69-1.21]$ & $0.82[0.61-1.10]$ \\
\hline Unemployed & $1.84^{* *}[1.22-2.78]$ & $1.18[0.76-1.82]$ & $1.16[0.74-1.80]$ & $1.31[0.83-2.07]$ \\
\hline Housewives & 1.07 [0.82-1.38] & $0.84[0.62-1.14]$ & $0.83[0.62-1.13]$ & $0.78[0.58-1.06]$ \\
\hline Family structure & $p^{9}<0.05$ & $p^{9}<0.05$ & $p^{g}<0.10$ & $p^{g}$ ns \\
\hline Child \& partner & 1.00 & 1.00 & 1.00 & 1.00 \\
\hline Only partner & $1.02[0.81-1.30]$ & $1.10[0.86-1.41]$ & $1.10[0.86-1.41]$ & $1.00[0.78-1.28]$ \\
\hline No children no partner & $1.00[0.78-1.27]$ & $1.02[0.78-1.33]$ & $1.06[0.81-1.38]$ & $1.06[0.81-1.40]$ \\
\hline Only children & $1.47^{* *}[1.13-1.90]$ & $1.46^{* *}[1.12-1.90]$ & $1.45^{* *}[1.11-1.89]$ & $1.35^{*}[1.02-1.78]$ \\
\hline Smoking & $\mathrm{p}^{9}<0.001$ & & $\mathrm{p}^{\mathrm{g}}<0.001$ & $p^{9}<0.01$ \\
\hline Never & 1.00 & & 1.00 & 1.00 \\
\hline In past & $1.27^{*}[1.01-1.58]$ & & $1.29^{*}[1.03-1.62]$ & $1.22^{\#}[0.97-1.54]$ \\
\hline Occasional & $1.32^{\#}[0.9-1.81]$ & & 1.25 [0.91-1.72] & $1.21[0.88-1.66]$ \\
\hline Current & $1.75^{* * *}[1.41-2.17]$ & & $1.58^{* * *}[1.26-1.98]$ & $1.51^{* * *}[1.20-1.90]$ \\
\hline BMI & $\mathrm{p}^{\mathrm{g}}<0.001$ & & $\mathrm{p}^{\mathrm{g}}<0.01$ & $\mathrm{p}^{\mathrm{g}}<0.05$ \\
\hline Under 20 & 0.97 [0.79-1.19] & & $0.96[0.78-1.18]$ & $0.92[0.75-1.13]$ \\
\hline $20-24.9$ & 1.00 & & 1.00 & 1.00 \\
\hline $25-29.9$ & $1.38^{*}[1.08-1.76]$ & & $1.33^{*}[1.04-1.69]$ & $1.36^{*}[1.07-1.75]$ \\
\hline $30+$ & $1.90^{* * *}[1.34-2.71]$ & & $1.69^{* *}[1.18-2.43]$ & $1.42^{\#}[0.96-2.09]$ \\
\hline Mental illness & $\mathrm{p}^{9}<0.001$ & & & $\mathrm{p}^{9}<0.001$ \\
\hline Yes vs. no & $4.48^{* * *}[2.38-8.41]$ & & & $7.55^{* * *}[3.61-15.78]$ \\
\hline Trauma \& musculoskeletal illness & $\mathrm{p}^{\mathrm{g}}<0.001$ & & & $\mathrm{p}^{9}<0.001$ \\
\hline Yes vs. no & $3.64^{* * *}[2.67-4.98]$ & & & $4.90^{* * *}[3.46-6.94]$ \\
\hline Cardiovascular illness & $\mathrm{p}^{9}<0.001$ & & & $\mathrm{p}^{\mathrm{g}}<0.001$ \\
\hline Yes vs. no & $4.02^{* * *}[2.73-5.93]$ & & & $4.86^{* * *}[3.21-7.38]$ \\
\hline Other diseases & $p^{9}<0.001$ & & & $\mathrm{p}^{9}<0.001$ \\
\hline Yes vs. no & $4.09^{* * * *}[3.24-5.16]$ & & & $5.53^{* * *}[4.28-7.16]$ \\
\hline \multirow[t]{2}{*}{ Language Russian vs. Estonian } & $\mathrm{p}^{\mathrm{g}}<0.001$ & $\mathrm{p}^{\mathrm{g}}<0.001$ & $\mathrm{p}^{\mathrm{g}}<0.001$ & $\mathrm{p}^{\mathrm{g}}<0.001$ \\
\hline & $2.51^{* * *}[2.05-3.07]$ & $2.12^{* * *}[1.72-2.61]$ & $2.05[1.66-2.53]$ & $1.92[1.55-2.38]$ \\
\hline
\end{tabular}

SEP = socioeconomic position; $\mathrm{BMI}=$ body mass index.

${ }^{a}$ All the associations were adjusted for language Russian vs. Estonian.

${ }^{\mathrm{b}}$ Model I SRH = age + one of the explanatory variables (education, personal income, employment status, family structure, BMI, smoking, chronic diseases).

cElaboration analysis by adding a group of explanatory variables in the model allows one to assess the mediation effect of the group of new variables.

${ }^{\mathrm{d}}$ Model II SRH = age + education + personal income + employment status + family structure (SEP variables).

${ }^{\mathrm{e}}$ Model III SRH $=$ age + SEP variables $+\mathrm{BMI}+$ smoking.

${ }^{f}$ Model IV SRH = age + SEP variables + behaviour variables + chronic diseases.

${ }^{9}$ Wald test for significance of the cumulative OR difference between the categories of the variable.

${ }^{\#} \mathrm{p}<0.1$; $\mathrm{p}<0.05 ;{ }^{* *} \mathrm{p}<0.01$; ${ }^{* * *} \mathrm{p}<0.001$; ns not significant. 
Table 5 Cumulative OR with $95 \% \mathrm{Cl}$ for self-rated health ( 1 = best health and $5=$ poorest health) by socioeconomic, behaviour factors and longstanding illness among women in Finland

\begin{tabular}{|c|c|c|c|c|}
\hline & Model I age-adjusted $^{a}$ & Model II + SEP adjusted ${ }^{\mathrm{b}, \mathrm{c}}$ & $\begin{array}{l}\text { Model III + behavior } \\
\text { risk factors }\end{array}$ & Model IV + chronic diseases ${ }^{b, e}$ \\
\hline Education & $p^{f}<0.001$ & $p^{f}<0.001$ & $p^{f}<0.001$ & $p^{f}<0.001$ \\
\hline$<10$ yrs & 1.00 & 1.00 & 1.00 & 1.00 \\
\hline $10-12$ yrs & $0.69^{\#}[0.46-1.02]$ & $0.78[0.52-1.16]$ & $0.83[0.56-1.24]$ & $0.87[0.58-1.30]$ \\
\hline $13-15$ yrs & $0.52^{* *}[0.36-0.76]$ & $0.60^{* *}[0.41-0.88]$ & $0.69^{\#}[0.47-1.01]$ & $0.77[0.52-1.14]$ \\
\hline $16+y r s$ & $0.35^{* * *}[0.23-0.52]$ & $0.40^{* * *}[0.27-0.60]$ & $0.47^{* * *}[0.31-0.72]$ & $0.51^{* *}[0.34-0.77]$ \\
\hline Personal income & $p^{f}<0.001$ & $p^{f}<0.01$ & $p^{f}<0.01$ & $p^{f}<0.01$ \\
\hline Lowest quartile & 1.00 & 1.00 & 1.00 & 1.00 \\
\hline 2nd quartile & $0.61^{* *}[0.46-0.81]$ & $0.68^{* *}[0.51-0.89]$ & $0.71^{*}[0.53-0.94]$ & $0.75^{\#}[0.56-1.00]$ \\
\hline 3rd quartile & $0.47^{* * *}[0.35-0.62]$ & $0.58^{* *}[0.43-0.79]$ & $0.60^{* *}[0.44-0.82]$ & $0.63^{* *}[0.46-0.86]$ \\
\hline Highest quartile & $0.51^{* * *}[0.38-0.68]$ & $0.74^{\#}[0.53-1.02]$ & $0.79[0.57-1.10]$ & $0.85[0.61-1.17]$ \\
\hline Employment & $p^{f}<0.001$ & $p^{f} n s$ & $p^{f} n s$ & $p^{f} n s$ \\
\hline Employed & 1.00 & 1.00 & 1.00 & 1.00 \\
\hline Student & $1.31^{\#}[0.96-1.78]$ & $1.19[0.85-1.67]$ & $1.26[0.90-1.77]$ & $1.20[0.86-1.69]$ \\
\hline Unemployed & $2.10^{* * *}[1.50-2.92]$ & $1.58^{*}[1.08-2.31]$ & $1.53^{*}[1.05-2.24]$ & $1.31[0.90-1.90]$ \\
\hline Housewives & $1.40^{*}[1.01-1.92]$ & $1.21[0.86-1.71]$ & $1.24[0.88-1.75]$ & $1.13[0.78-1.63]$ \\
\hline Family structure & $\mathrm{p}^{\mathrm{f}}<0.10$ & $p^{f} n s$ & $p^{f} n s$ & $p^{f}<0.05$ \\
\hline Child \& partner & 1.00 & 1.00 & 1.00 & 1.00 \\
\hline Only partner & $1.04[0.81-1.34]$ & $1.12[0.85-1.47]$ & $1.13[0.86-1.48]$ & $1.17[0.89-1.53]$ \\
\hline No children no partner & $0.95[0.71-1.26]$ & $0.91[0.67-1.24]$ & $0.84[0.62-1.15]$ & $0.80[0.58-1.10]$ \\
\hline Only children & $1.50^{*}[1.08-2.09]$ & $1.24[0.89-1.73]$ & $1.26[0.89-1.76]$ & $1.29[0.90-1.84]$ \\
\hline Smoking & $p^{f}<0.001$ & & $p^{f}<0.01$ & $p^{f}<0.001$ \\
\hline Never & 1.00 & & 1.00 & 1.00 \\
\hline In past & $1.05[0.81-1.36]$ & & $0.92[0.70-1.21]$ & $0.95[0.72-1.25]$ \\
\hline Occasional & $0.61^{*}[0.38-0.99]$ & & $0.56^{*}[0.34-0.92]$ & $0.50^{* *}[0.30-0.84]$ \\
\hline Current & $1.60^{* * *}[1.29-1.98]$ & & $1.29^{*}[1.02-1.62]$ & $1.32^{*}[1.04-1.67]$ \\
\hline BMI & $p^{f}<0.001$ & & $p^{f}<0.001$ & $p^{f}<0.001$ \\
\hline Under 20 & $1.27^{\#}[0.97-1.67]$ & & $1.19[0.90-1.57]$ & $1.21[0.92-1.58]$ \\
\hline $20-24.9$ & 1.00 & & 1.00 & 1.00 \\
\hline $25-29.9$ & $1.40^{* *}[1.13-1.75]$ & & $1.34^{*}[1.07-1.68]$ & $1.34^{*}[1.06-1.70]$ \\
\hline $30+$ & $3.02^{* * * *}[2.28-4.02]$ & & $2.85^{* * *}[2.13-3.81]$ & $2.36^{* * *}[1.75-3.18]$ \\
\hline Mental illness & $p^{f}<0.001$ & & & $p^{f}<0.001$ \\
\hline Yes vs. no & $10.4^{* * *}[5.86-19.15]$ & & & $10.90^{* * *}[5.41-21.96]$ \\
\hline Trauma \& musculoskeletal & $p^{f}<0.001$ & & & $\mathrm{p}^{\mathrm{f}}<0.001$ \\
\hline Yes vs. no & $4.36^{* * *}[3.26-5.83]$ & & & $4.57^{* * *}[3.34-6.25]$ \\
\hline Cardiovascular illness & $p^{f}<0.001$ & & & $p^{f}<0.001$ \\
\hline Yes vs. no & $3.76^{* * *}[2.17-6.52]$ & & & $3.02^{* * *}[1.72-5.31]$ \\
\hline Other diseases & $p^{f}<0.001$ & & & $p^{f}<0.001$ \\
\hline Yes vs. no & $1.82^{* * *}[1.44-2.30]$ & & & $2.59^{* * *}[1.98-3.39]$ \\
\hline
\end{tabular}

${ }^{a}$ Model I SRH = age + one of the explanatory variables (education, personal income, employment status, family structure, BMI, smoking, chronic diseases).

${ }^{b}$ Elaboration analysis by adding a group of explanatory variables in the model allows one to assess the mediation effect of the group of new variables.

${ }^{c}$ Model II SRH = age + education + personal income + employment status + family structure (SEP variables).

${ }^{\mathrm{d}}$ Model III SRH $=$ age + SEP variables $+\mathrm{BMI}+$ smoking.

${ }^{\mathrm{e}}$ Model IV SRH = age + SEP variables + behaviour variables + chronic diseases.

${ }^{f}$ Wald test for significance of the cumulative OR difference between the categories of the variable.

${ }^{\#} \mathrm{p}<0.1 ;{ }^{*} \mathrm{p}<0.05$; ${ }^{* *} \mathrm{p}<0.01$; ${ }^{* * *} \mathrm{p}<0.001$; ns not significant.

$\mathrm{SEP}=$ socioeconomic position. 


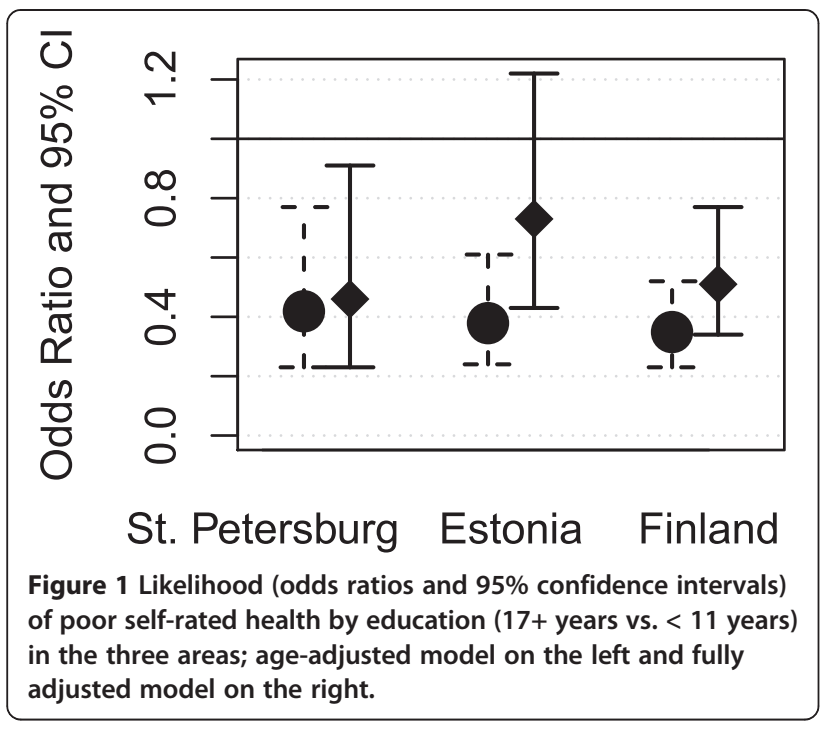

In St. Petersburg unlike the other two areas, housewives rather than employed women had less often poor $\mathrm{SRH}(\mathrm{COR}=0.66)$. The better SRH of housewives in St. Petersburg remained in the fully adjusted model (Figure 3). The interaction between country and employment status was not significant (data not shown).

Mutual adjustment for the socioeconomic position variables revealed marginally significant interaction between country and employment status, with the interaction between country and income remaining significant and the interaction between country and education remaining insignificant (data not shown).

Health behaviour and differences in socioeconomic self-rated health

The expected associations between health behaviour factors (BMI and smoking) and poor self-rated health was

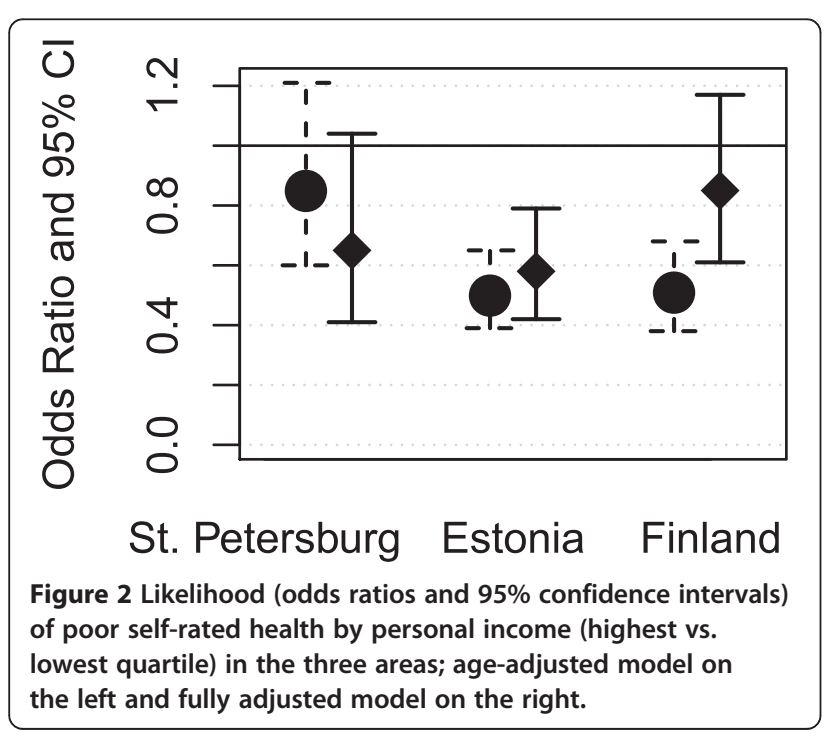

found in all studied populations (age-adjusted models in Tables 3, 4 and 5). Current smokers had poorer self-rated health in comparison to never smokers. The overweight and obese respondents were more likely to report poorer health in comparison to those with normal weight.

Adjustment for smoking and BMI (Model III vs. Model I) reduced the negative association between education $(17+$ years vs. $<11$ years $)$ and poorer health by $7 \%, 44 \%$ and $18 \%$ among St. Petersburg, Estonian and Finnish women, respectively; however, the association between education and health remained significant. Adjustment for health behaviour did not affect the interaction between income and country, although it reduced the significance of the interaction between employment status and country (data not shown).

\section{Longstanding illnesses and differences in socioeconomic self-rated health}

A positive association was found between longstanding illness and poorer self-rated health (age-adjusted models in Tables 3, 4 and 5). Adjustment for chronic limiting conditions reduced the educational health gradient further (Model III vs. Model IV) by 23\% for highest (17+ years) vs. lowest ( $<11$ years) education among Estonians, and among Finns by $8 \%$, although among Russians the association remained unchanged. The income health gradient was reduced in Finns and Estonians. Also the incorporation of chronic diseases in the explanatory model reduced the association between unemployment and poorer SRH among Finns, and the association lost its significance.

The interaction between income and country remained significant, $\mathrm{p}<0.05$, but the modification effect of country on the association between education/employment status and health remained insignificant (data not shown).

\section{Discussion}

In all the studied populations education was associated with SRH; this finding is in accordance with previously reported results from surveys conducted in the former Soviet Union and also in other European countries $[34,35]$. However, in respect to material economic factors, we found neither a mediating effect of income or employment on the association between education and health nor an independent income-health gradient in St. Petersburg comparable to that found in Finnish and Estonian women. The finding is consistent with the previously reported lack of an explanatory effect of income on the association between education and mortality in Russian women and a relatively weak correlation between income and mortality [36]. It was emphasized that in the Russian settings in the 1990s, inflation, bartering, living on savings, along with a low economic return for education may have distorted the link between income 


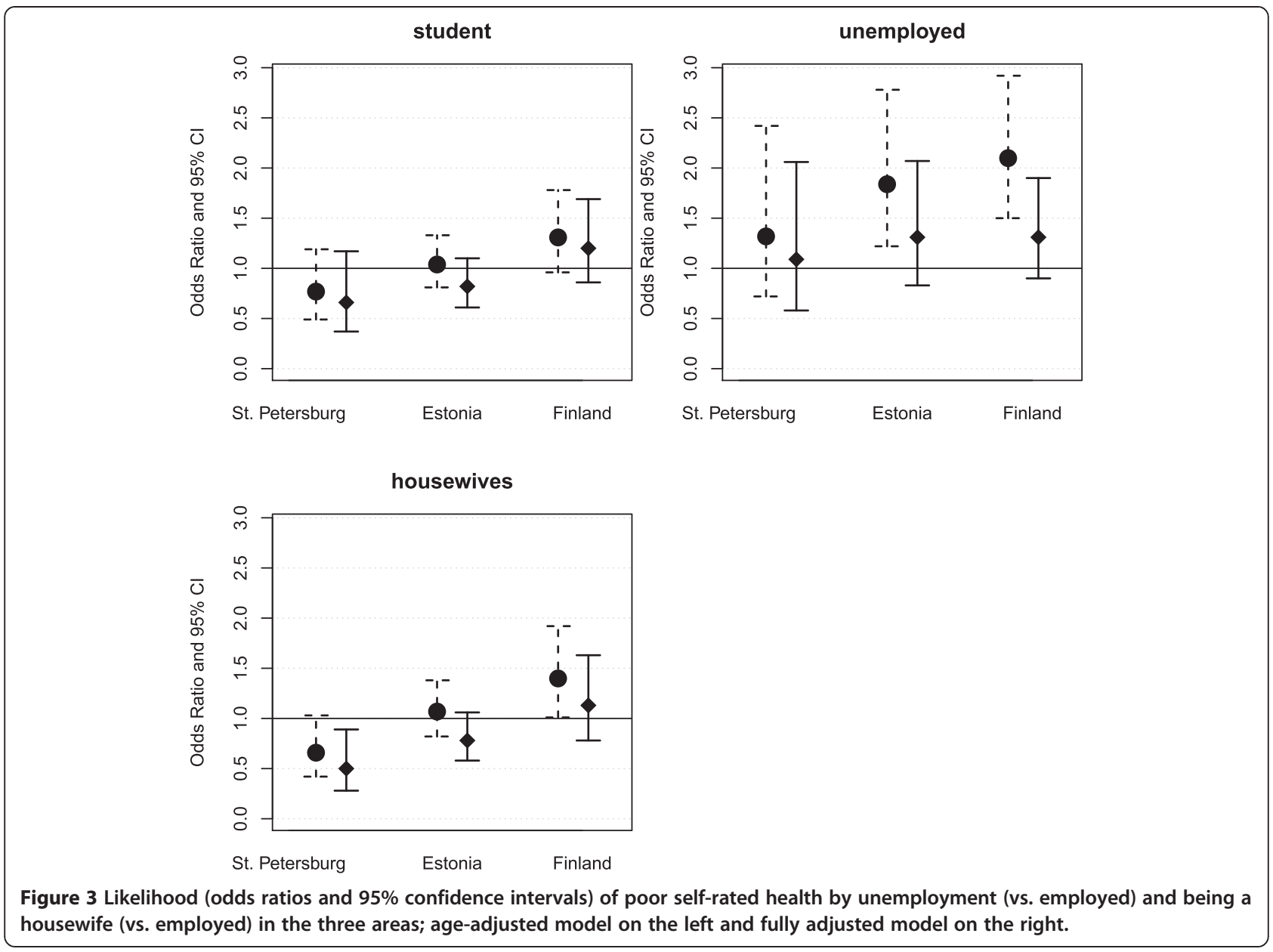

and health that has been consistently reported in other industrialized countries [36]. Our findings are in line with those statements. For that reason, education rather than income could be considered as a meaningful dimension of women's socioeconomic position in St. Petersburg.

We did not examine the influence of household income on women's health, which could act as a buffer for the financial difficulties of those with low individual income. This was partly due to anticipated difficulty in interpreting family income, as the concept of family varies between the study areas. In St. Petersburg extended families as economic units were still common and in Finland common law marriages were very common; also taxation is separate for the woman and her partner.

In St. Petersburg housewives rather than employed women had better perceived health, unlike in the two other areas. We do not know what this finding reflects, but it could relate to selection to become a housewife by area.

In all three studied populations the association between socioeconomic position and health was partially explained by smoking habits and relative body weight. This finding suggests that in St. Petersburg lifestyle factors could be mediating factors in the association between social status and perceived health in the same way as in Estonia and Finland. However, it is worth noticing that a selection process could also be involved. In Finland it has been shown that smoking in adolescence predicts poorer educational achievements and affects future socioeconomic status [37]. Thus smoking may act as a factor of indirect selection for the association between education and health and not only mediate, but confound the association between education and health. Psychosocial stress, resulting from disadvantaged socioeconomic position, may affect risk-taking behaviour such as smoking and at the same time cause poor health. Due to this mechanism the contribution of the behavioural factors to the educational health divide may be overestimated.

Limiting longstanding illness may affect the association between socioeconomic position and health via mediating and selecting mechanisms. Unlike in Estonia and Finland, adjustment for longstanding illness did not affect the educational health gradient among women from St. Petersburg. A lower rate of health care utilisation in the lower educational categories [38] may also result in misreporting of longstanding illness by those with poorer education. 
Our findings do not allow for a direct formulation of appropriate interventions targeting lower educated women for lifestyle modification so as reduce the educational health divide. However, they show that there may be a need to tailor intervention designs for different countries and areas. Future research regarding such interventions and identification of the potential barriers to healthy choices among women with low socioeconomic position are needed.

\section{Study strengths and limitations}

Strengths of this comparative study include that all surveys were population based and done in close-by years. In Finland the survey was made three years earlier than in the other countries. But there were no major economic or social changes between 2001 and 2004. Surveys were carried out in the context of larger surveys that did not focus on socioeconomic differences, which may reduce any potential reporting bias by the studied question. The questions used were very similar across the studies. The Finnish survey was used as a model and the Estonian and Russian questionnaires were each compiled by the research team with the aim of achieving good comparability, regardless of the different language structures. Furthermore, local researchers were strongly involved in negotiating and arriving at the appropriate word meanings.

The study limitations are those typical of questionnaires and self-reporting: the cross-sectional study design cannot provide evidence on causality; the effect of selection bias cannot be estimated; and presumably a lower participation rate for marginalized groups may lead to underestimation of the contribution of lifestyle factors to health disparities. Furthermore, the response rates were different in the study countries and this may weaken comparisons across countries.

Even though the question on self-rated health was the same in all study areas, it is possible that the meaning of the question and the answer options varied by country due to language and cultural differences. In Finland, data of body weight and height were measured and data of income obtained from tax records. In St. Petersburg and Estonia they were asked from the respondents, and the answers may be less accurate than in Finland. Getting data from tax offices was not possible in St. Petersburg and Estonia, and data collection method did not allow direct measurements.

\section{Abbreviations \\ BMl: Body mass index; OR: Odds ratio; SRH: Self-rated health; \\ COR: Cumulative odds ratio.}

\section{Competing interests}

The authors declare that they have no competing interests.

\section{Authors' contributions}

TD participated in the data collection (St. Petersburg), originated the idea, planned the analysis and prepared the draft manuscript. TH carried out the analysis and commented the manuscript. ER participated in the data collection (St. Petersburg), finalized the article and commented the manuscript. EH participated in designing the study and commented the manuscript. EH-M participated in designing the study and commented the manuscript. ML participated in the data collection (Estonia) and commented the manuscript OK participated in designing the study and commented the manuscript. SK participated in the data collection (Finland), designing the study and commented the manuscript. All authors read and approved the final manuscript.

\section{Acknowledgements}

We would like to thank Kaja Rahu for her valuable work in data management.

\section{Funding}

The study was financially supported by the Academy of Finland (208180, 208186), the Russia in Flux programme (208180 and 208186) and the Baltic Sea Task Force (PH030). The survey in Estonia was financially supported by the Estonian Science Foundation (5456), the Academy of Finland, the Estonian Ministry of Education and Science (SF0182641s04 and SF940026s07) and the Baltic Sea Task Force.

\section{Author details}

${ }^{1}$ St. Petersburg Medical Academy of Postgraduate Studies or North-Western State Medical University named after I.I. Mechnikov, 193015 Kirochnaja ul. 41, St. Petersburg, Russia. ${ }^{2}$ The National Institute for Health and Welfare (THL), P. O. Box 30, Helsinki FI-00271, Finland. ${ }^{3}$ Department of Social Research, University of Helsinki, Box 24, Helsinki 00014, Finland. ${ }^{4}$ Department of Obstetrics and Gynaecology, University of Tartu, L. Puusepa 8, Tartu 50406, Estonia.

Received: 10 October 2013 Accepted: 6 May 2014

Published: 17 May 2014

\section{References}

1. Rahkonen O, Laaksonen M, Martikainen P, Roos E, Lahelma E: Job control, job demands, or social class? The impact of working conditions on the relation between social class and health. J Epidemiol Community Health 2006, 60:50-54.

2. Kestilä L, Martelin T, Rahkonen $\mathrm{O}$, Härkänen $\mathrm{T}$, Koskinen $\mathrm{S}$ : The contribution of childhood circumstances, current circumstances and health behaviour to educational health differences in early adulthood. BMC Public Health 2009, 9:164.

3. Nieminen T, Martelin T, Koskinen S, Aro H, Alanen E, Hyyppa MT: Social capital as a determinant of self-rated heath and psychological well-being. Int J Public Health 2010, 55:531-542.

4. Kostiainen E, Martelin T, Kestila L, Martikainen P, Koskinen S: Employee, partner and mother: woman's three roles and their implications for health. J Fam Issues 2009, 30:1122-1150.

5. Ben-Shlomo Y, Kuh D: A life-course approach to chronic disease epidemiology: conceptual models, empirical challenges and interdisciplinary perspectives. Int J Epidemiol 2002, 31:285-293.

6. Kestilä L, Martelin T, Rahkonen O, Joutsenniemi K, Pirkola S, Poikolainen K, Koskinen S: Childhood and current determinants of heavy drinking in early adulthood. Alcohol 2008, 43:460-469.

7. Farmer MM, Ferraro KF: Distress and perceived health: mechanisms of health decline. J Health Soc Behav 1997, 38:298-311.

8. Lundberg O, Manderbacka K: Assessing reliability of a measure of self-rated health. Scand J Soc Med 1996, 24:218-224.

9. Miilunpalo S, Vuori I, Oja P, Pasanen M, Urponen H: Self-rated health status as a health measure: the predictive value of self-reported health status on the use of physician services and on mortality in the working-age population. J Clin Epidemiol 1997, 50:517-528

10. Carlson P: Risk behaviour and self-rated health in Russia 1998. J Epidemiol Community Health 2001, 55:806-817.

11. Heistaro S, Laatikainen T, Vartiainen E, Puska P, Uutela A, Pokusaeva S, Uhanov M: Self-reported health in the republic of Karelia, Russia and in North Karelia, Finland in 1992. Eur J Public Health 2001, 11:74-80.

12. Palosuo H, Uutela A, Zhuravleva I, Lakomova N: Social patterning of ill health in Helsinki and Moscow. Results from a comparative survey in 1991. Soc Sci Med 1998, 46:1121-1136. 
13. Carlson P: Educational differences in self-rated health during the Russian transition. Evidence from Taganrog 1993-1994. Soc Sci Med 2000, 51:1363-1374

14. Rojas Y, Carlson P: The stratification of social capital and its consequences for self-rated health in Taganrog, Russia. Soc Sci Med 2006, 62:2732-2741.

15. Nicholson A, Bobak M, Murphy M, Rose R, Marmot M: Socio-economic influences on self-rated health in Russian men and women - a life course approach. Soc Sci Med 2005, 61:2345-2354.

16. Lundberg J, Bobak M, Malyutina S, Kristenson M, Pikhart H: Adverse health effect of low levels of perceived control in Swedish and Russian community samples. BMC Public Health 2007, 7:314.

17. Nazarova I: Self-rated health and occupational conditions in Russia. Soc Sci Med 2000, 51:1375-1385.

18. Cockerham WC: Health lifestyles and the absence of the Russian middle class. Sociol Health IIIn 2007, 29:457-473.

19. Cockerham WC: Health lifestyles and political ideology in Belarus, Russia and Ukraine. Soc Sci Med 2006, 62:1799-1809.

20. Davis CE, Deev AD, Shestov DB, Perova NV, Plavinskaya SE, Abolafia JM: Correlates of mortality in Russian and US women. The Lipid Research Clinics Program. Am J Epidemiol 1994, 139:369-379.

21. Malytina S, Bobak M, Simonova G, Gafarov V, Nikitin Y, Marmot M: Education, marital status, and total and cardiovascular mortality in Novosibirsk, Russia: a prospective cohort study. Ann Epidemiol 2004, 14:244-249.

22. Health for All database. http://data.euro.who.int/hfadb/.

23. Estonian women are in between Eurostat. http://epp.eurostat.ec.europa. eu/tgm/refreshTableAction.do?tab=table\&plugin $=1 \&$ pcode $=\operatorname{tsdph} 210$ \&language $=e n$.

24. Umberson D: Family status and health behaviors: social control as a dimension of social integration. J Health Soc Behav 1987, 28:306-319.

25. Nieminen T, Prättälä R, Martelin T, Härkänen T, Hyyppä MT, Alanen E, Koskinen S: Social capital, health behaviours and health: a population-based associational study. BMC Public Health 2013, 13:613.

26. Dahlin J, Härkönen J: Cross-national differences in the gender gap in subjective health in Europe: Does country-level gender equality matter? Soc Sci Med 2013, 98:24-28.

27. Pärna K, Ringmets I: Comparison of socioeconomic differences in self-perceived health in Estonia and Finland. Scand J Public Health 2010, 38(2):129-134.

28. Regushevskaya E, Dubikaytis T, Laanpere M, Nikula M, Kuznetsova O, Haavio-Mannila E, Karro H, Hemminki E: Risk factors for induced abortions in St Petersburg, Estonia and Finland. Results from the surveys among women of reproductive age. Eur J Contracept Reprod Health Care 2009, 3:176-186.

29. Kesseli K, Regushevskaya E, Doubikaytis T, Kirichenko S, Rotkirch A, Haavio-Mannila E, Kuznetsova O, Hemminki E: Reproductive Health and Fertility in St. Petersburg 2004- Report on a Survey of 18-44 Year old Women in 2004. In Working papers 60. Helsinki: Department of Sociology, University of Helsinki; 2005.

30. Part K, Laanpere M, Rahu K, Haldre K, Rahu M, Karro H: Estonians women's Health: Sexual and Reproductive Health, Health Behaviour, Attitudes and use of Health Services. Survey Report. Tartu, Estonia: University of Tartu; 2007. http://www.ut.ee/ARNS/Eesti_naise_tervis1.pdf.

31. Aromaa A, Koskinen S: Health and Functional Capacity in Finland. Baseline Results of the Health 2000. Health Examination Survey. Helsinki: Publications of the National Public Health Institute B12/2004; 2004.

32. Heistaro S: Methodology Report Health 2000 Survey. Publications of the National Public Health Institute, KTL B 26/2008; http://www.terveys2000.fi/ doc/methodologyrep.pdf.

33. Graubard Bl, Korn EL: Predictive margins with survey data. Biometrics 1999 , 55:652-659

34. Bobak M, Pikhart H, Rose R, Hertzman C, Marmot M: Socioeconomic factors, material inequalities, and perceived control in self-rated health: cross-sectional data from seven post-communist countries. Soc Sci Med 2000, 51:1343-1350.

35. Eikemo TA, Bambra C, Joyce K, Dahl E: Welfare state regimes and income-related health inequalities: a comparison of 23 European countries. Eur J Public Health 2008, 18:593-599.

36. Perlman F, Bobak M: Socioeconomic and behavioral determinants of mortality in posttransition Russia: a prospective population study. Ann Epidemiol 2008, 18:92-100
37. Koivusilta L, Rimpelä A, Rimpelä M: Health related lifestyle in adolescence predicts adult educational level: a longitudinal study from Finland. J Epidemiol Community Health 1998, 52:794-801

38. Perlman F, Balabanova D, McKee M: An analysis of trends and determinants of health insurance and healthcare utilisation in the Russian population between 2000 and 2004: the 'inverse care law' in action. BMC Health Serv Res 2009, 9:68.

doi:10.1186/1475-9276-13-39

Cite this article as: Dubikaytis et al.: Socioeconomic differences in self-rated health among women: a comparison of St. Petersburg to Estonia and Finland. International Journal for Equity in Health 2014 13:39.

\section{Submit your next manuscript to BioMed Central and take full advantage of:}

- Convenient online submission

- Thorough peer review

- No space constraints or color figure charges

- Immediate publication on acceptance

- Inclusion in PubMed, CAS, Scopus and Google Scholar

- Research which is freely available for redistribution 